\title{
Erratum to: Shame on You: When Materialism Leads to Purchase Intentions Toward Counterfeit Products
}

\author{
Alexander Davidson $^{1} \cdot$ Marcelo Vinhal Nepomuceno $^{2} \cdot$ Michel Laroche $^{1}$ (i)
}

Published online: 24 May 2017

(C) Springer Science+Business Media Dordrecht 2017

\section{Erratum to: J Bus Ethics}

\section{DOI 10.1007/s10551-017-3479-5}

In this article the name of the second author is Marcelo Vinhal Nepomuceno. The original article has been corrected. doi:10.1007/s10551-017-3479-5.

Michel Laroche

michel.laroche@concordia.ca

Alexander Davidson

alex.davidson@concordia.ca

Marcelo Vinhal Nepomuceno

marcelo.nepomuceno@hec.ca

1 Concordia University, 1455 de Maisonneuve Blvd. West, Montreal, QC H3G 1M8, Canada

2 HEC Montreal, 3000 chemin de la Côte-Sainte-Catherine (office: 4.756), Montreal, QC H3T 2A7, Canada 